\title{
Alignment-induced reconfigurable walls for patterning and assembly of liquid crystal skyrmions
}

\author{
Ayhan Duzgun* and Avadh Saxena $\odot$ \\ Theoretical Division and Center for Nonlinear Studies, Los Alamos National Laboratory, Los Alamos, New Mexico 87545, USA \\ Jonathan V. Selinger (1) \\ Advanced Materials and Liquid Crystal Institute and Department of Physics, Kent State University, Kent, Ohio 44242, USA
}

(Received 29 July 2020; revised 1 November 2020; accepted 18 December 2020; published 15 January 2021)

\begin{abstract}
Skyrmions have attracted rapidly growing interest due to their topological properties and unique aspects for potential novel applications such as data storage and soft robotics. They can also serve as key elements for materials by design, self-assembly, and functional soft materials. While not real particles, these skyrmions behave like particles - they interact with each other and can be actuated by means of electric field, surface anchoring, and light. On the other hand, they are field configurations which have properties not possessed by real particles. Here we show that, by means of alignment-induced attractive and repulsive walls, skyrmions in chiral nematic liquid crystals can be precisely controlled and programed to serve as suitable building blocks for the realization of the above goals. Our work may stimulate new experimental efforts and concomitant applications in this direction.
\end{abstract}

DOI: 10.1103/PhysRevResearch.3.L012005

Introduction. Richard Feynman pointed out that control of things on a small scale would have great impact on customizing material properties and hence advance nanotechnology [1]. In this Letter, we propose that walls produced by imposing alignment patterns can enable individual control of liquid crystal (LC) skyrmions, along the line of such purposes: Precise control of skyrmions in a LC cell can be achieved and employed to realize designer materials with desired properties at the microscale.

Skyrmions are particle-like topological field configurations which were originally proposed as field configurations in particle physics [2], and later their realization in magnetic systems attracted much attention and extensive research has uncovered many complex features of magnetic skyrmions $[3,4]$. More recently, LC skyrmions have been realized as micron sized solitons in a chiral nematic material confined between two parallel substrates [5-10]. See Figs. 1(a)-1(c).

In LCs, full skyrmions are suitable for individual design and manipulation due to their particle-like properties; they exist as isolated objects [8] and, when not tilted, they interact with other skyrmions like local soft repulsive particles [11,12] unlike other structures such as merons (half-skyrmions) that are accompanied by topological defects and are generally reported to exist as lattices [13-17]. Additional properties lacked by real particles provide an even richer set of tools as skyrmions can be generated and decimated at will, are flexible and readily deformable, and their interaction can be switched between isotropic-repulsive and directional-attractive [11].

\footnotetext{
*ayhan@lanl.gov

Published by the American Physical Society under the terms of the Creative Commons Attribution 4.0 International license. Further distribution of this work must maintain attribution to the author(s) and the published article's title, journal citation, and DOI.
}

Novel mechanisms such as oscillating electric fields have been shown to drive particle-like excitations, for example static skyrmions [18] and solitary waves [19,20]. However, more localized control will better serve the purpose of design at the constituent level. In this work, we introduce a different approach enabling more precise and localized control and assembly of skyrmions into molecules: Instead of applying fields over a broad region, we generate regions with preferred alignment directions which act as repulsive or attractive walls. We impose the alignment of LC molecules by means of surface anchoring, electric field, and light. The reason we count light as a way to impose alignment is the following. Dimensionless alignment strength can be expressed as the ratio of anchoring and field coupling coefficients to the natural twist $q_{0}=2 \pi / p$, where $p$ is the helical pitch of the chiral nematic LC [see Eq. (1) and the section Model]. Thus reducing $q_{0}$ increases the resulting alignment strength. For example, in the experiments of Sohn et al. [21], light is used to push skyrmions out of the exposed region as light chemically reduces the natural twist. Our numerical simulations provide confirmation that modeling light exposure as a reduction in $q_{0}$ reproduces the observed behavior. More generally, we illustrate that any mechanism that impacts the helical pitch of the LC material can be used to generate attractive or repulsive regions.

We start by exploring the range of parameter space, as a reference point, in which we can realize skyrmions and then show how that range can be escaped to decimate skyrmions (a useful tool for dynamic manipulation). Next, we introduce repulsive and attractive walls produced by out of plane alignment and demonstrate how these soft and hard walls (obstacles) can be employed to guide and move skyrmions. Finally, we present simulations accompanied by an analytical calculation in the Supplemental Material (SM) [22] to illustrate that obstacles with dynamically controllable size can be achieved when voltage across small electrodes is used as the 


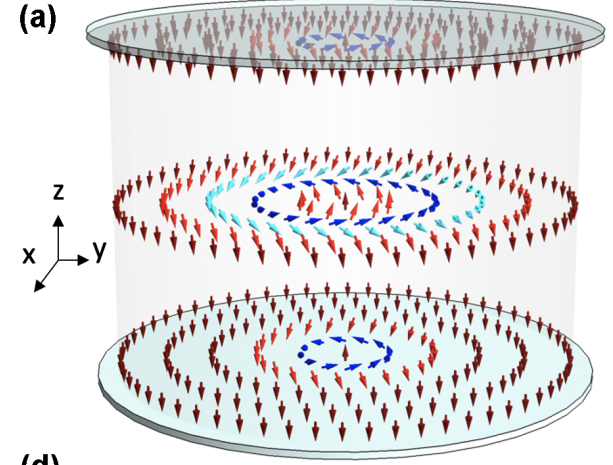

(d)

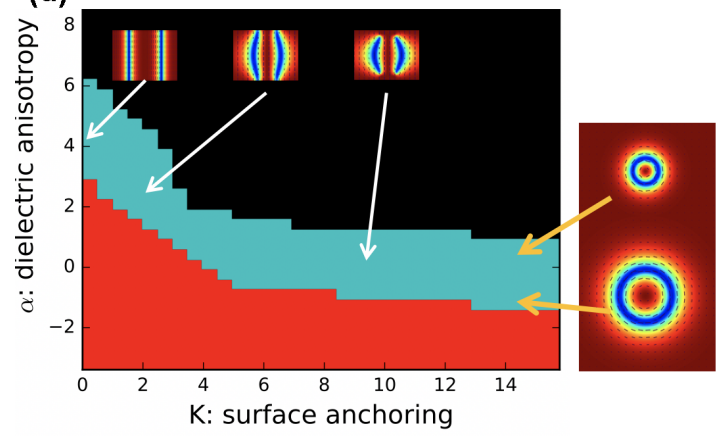

(b)

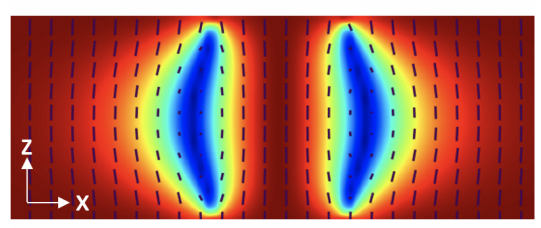

(c)

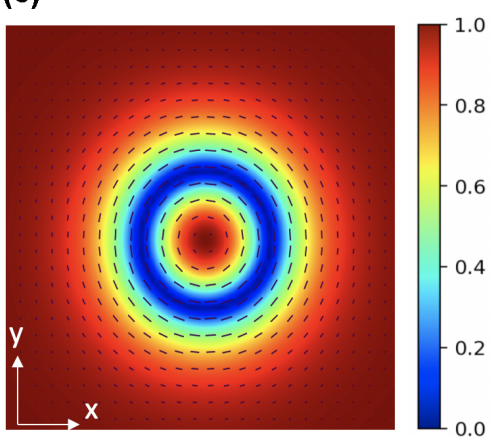

(e)

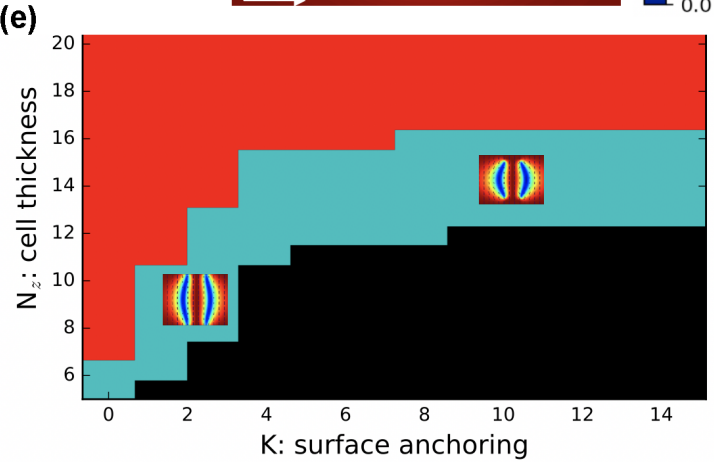

FIG. 1. Skyrmions realized in a chiral LC host confined between two parallel plates. (a) Vectorized director configuration of a skyrmion on three planes. (b) Vertical and (c) horizontal cross sections of a skyrmion. Color mapping indicates the vertical component $\left|n_{z}\right|$ and black dashed lines indicate the projection of the director on the plane shown. (d) For the cell thickness $N_{z}=15$, field vs. surface anchoring phase diagram. (e) For no field ( $\alpha=0$ ) cell thickness vs. surface anchoring phase diagram of skyrmions. [(d) and (e)] As the background alignment is varied, skyrmions exist between vertical nematic and cholesteric phases. The unit of $K$ is $K_{0}=L S q_{0}$ and the unit of $\alpha$ is $\alpha_{0}=L S q_{0}^{2}$. The unit of cell thickness is one lattice spacing equal to $1 / 40$ of the cholesteric pitch $p=2 \pi / q_{0}$.

wall generation mechanism. The size of these obstacles can be reduced all the way to zero by tuning the voltage, therefore this method allows even more precise and individual control over skyrmions.

Here we emphasize that these mechanisms, due to their nature, can be best understood by viewing the videos included in the SM [22]. This Letter investigates the case of upright skyrmions stabilized by vertical alignment and lays the foundation for future work, in which we aim to extend our analyses to the case of topologically equivalent tilted skyrmions [23].

Model. Consider an LC cell consisting of a chiral nematic LC confined between two parallel plates. We use $Q$-tensor representation to describe the average orientation of LC molecules at a certain position which is defined as $Q_{\alpha \beta}=\frac{3}{2}\left(\left\langle n_{\alpha} n_{\beta}\right\rangle-\frac{1}{3} \delta_{\alpha \beta}\right)$, where $n_{\alpha}$ are the components of the director field. In this notation Landau-de Gennes type free energy per unit volume, with single constant approximation, can be expressed as

$$
\begin{aligned}
f= & \frac{1}{2} a \operatorname{Tr}\left(Q^{2}\right)+\frac{1}{3} b \operatorname{Tr}\left(Q^{3}\right)+\frac{1}{4} c\left[\operatorname{Tr}\left(Q^{2}\right)\right]^{2} \\
& +\frac{1}{2} L\left(\partial_{\gamma} Q_{\alpha \beta}\right)\left(\partial_{\gamma} Q_{\alpha \beta}\right)-2 q_{0} L \epsilon_{\alpha \beta \gamma} Q_{\alpha \rho} \partial_{\gamma} Q_{\beta \rho} \\
& -\left\{\Delta \epsilon E^{2}+K\left[\delta(z)+\delta\left(z-N_{z}\right)\right]\right\} Q_{z z} .
\end{aligned}
$$

The first line above is the thermal part. Below a critical temperature embedded in $a$, it yields a nematic phase with scalar order parameter $S$ determined by $a, b$, and $c$. The second line is the elastic part with coefficient $L$ and favors a natural twist $q_{0}$. Here $S$ is approximately constant everywhere except in defect regions.
Alignment is achieved via the third line which represents the effect of an external electric field $E$ and the homeotropic surface anchoring at the confining surfaces $(z=0$ and $z=$ $N_{z}$ ). For simplicity, the field is assumed to be uniform and pointing in the $z$ direction. With the substitution of $\alpha=\Delta \epsilon E^{2}$, where $\Delta \epsilon$ is the dielectric anisotropy, positive values of $\alpha$ indicate a material with easy-axis alignment while negative values indicate easy-plane alignment. As for the surface anchoring, $K$ is the coupling coefficient between $Q$ and a perfect alignment in the $z$ direction which enforces easy-axis alignment along $z$. The unit of $\alpha$ will be expressed in terms of $\alpha_{0}=L S q_{0}^{2}$ and the unit of $K$ in terms of $K_{0}=L S q_{0}$ so that they provide a comparison of the cholesteric pitch $p=2 \pi / q_{0}$ to the electric coherence length $\sqrt{L S / \alpha}$ and the extrapolation length $K / L S$, respectively. Larger values of $K / N_{z}$ and $\alpha$ represent greater unwinding strength against the natural twist $q_{0}$. Reduction of $q_{0}$ (light exposure) leads to greater $K / K_{0}$ and $\alpha / \alpha_{0}$ values and hence to a stronger alignment effect.

Numerical solution to the above free energy equation is obtained by simulations with overdamped relaxation dynamics, which is detailed in SM [22].

Isolated skyrmions. A strong easy-axis alignment yields a vertical nematic phase. With weaker alignment, a cholesteric phase is observed. An intermediate range of alignment strength allows isolated skyrmions with director profiles depicted in Figs. 1(a)-1(c) $[9,10,16,24-26]$. The alignment strength is controlled by combinations of $K, N_{z}$, and $\alpha$. Figures 1(d) and 1(e) show $K-\alpha$ phase diagram for $N_{z}=15$ and 
$K-N_{z}$ phase diagram for $\alpha=0$. To investigate the skyrmion behavior, we embed a generic skyrmion director profile inside a uniform background and let the system relax. If the alignment is too strong, then the skyrmion shrinks and disappears. If the alignment is too weak, then it expands and breaks into cholesteric stripes. The right panel of Fig. 1(d), shows how the skyrmion size is controlled as the background field is varied.

Three-dimensional (3D) director profiles of the skyrmions vary depending on the alignment strength and mechanism. As seen in the insets of Fig. 1(d), if field alone is used, then a $z$-invariant structure forms. As $K$ is increased, the skyrmion takes a barrel shape (spherulite) and finally point or loop defects are formed. This behavior and further details have been discussed by Tai et al. [26].

The behavior illustrated here is key to generating and decimating skyrmions at will in any desired (programed) fashion. Skyrmions written by laser light [8] can be decimated by locally escaping the suitable parameter range.

Walls produced by strong vertical alignment. The director profile of a skyrmion on a vertical cross section is basically a kink from its center to periphery in any radial direction as shown in Fig. 2(a) where the green line indicates the absolute value of $n_{z}$. For an isolated skyrmion embedded in a uniform far field, the $180^{\circ}$ rotation of the polar angle from the center to the periphery takes place over a characteristic length scale. That length, determined by the natural twist coupled with the frustration in the system, is roughly a measure of how far away the skyrmion prefers to be from the perfectly vertical far field. Therefore, if we enforce a vertical alignment in a region, then we will essentially generate a wall that will push the skyrmions away. It is also clear that particle-like repulsion of two skyrmions is due to the same reason-optimum relaxation length of the gradients of the polar angle to the minimum energy configuration.

Now we investigate the range of interaction between a skyrmion and a hard wall (produced by a very strong field) by placing a skyrmion near the wall and letting the system relax. In Figs. 2(b) and 2(c), we plot the ratios $x / p, x / R$, and $(x-R) / p$ vs. time for $2 \mathrm{D}$ and $3 \mathrm{D}$ skyrmions of various sizes, where $x$ is the distance from the center of the skyrmion to the wall and $R$ is defined as the distance from the skyrmion center over which the first $90^{\circ}$ rotation of the polar angle occurs (radius of the blue circle). Thus the rotation from $90^{\circ}$ to $180^{\circ}$ occurs over $x-R$ because the hard wall maintains vertical alignment on the boundary. The size of the skyrmions is controlled by varying the alignment strength. In the case of a 2D skyrmion, we set $K=0$ and vary $\alpha$. In the case of a 3D skyrmion, we set $\alpha=0$ and vary $K$ (see the SM [22] for the parameters used.)

The results illustrate that the gradients of the polar angle are asymmetric around the horizontal direction. Furthermore, while the distance of rotation from $0^{\circ}$ to $90^{\circ}(R)$ varies greatly with the alignment strength, the rotation from $90^{\circ}$ to $180^{\circ}$ interestingly takes place over an almost constant distance $x-R$, which stays near $0.3 p$ for $2 \mathrm{D}$ and $0.5 p$ for $3 \mathrm{D}$ skyrmions. Therefore, the range of interaction $x$ is not a certain multiple of $R$ but varies from two to several skyrmion sizes $(R)$.

Repulsive or attractive regions. Confinement and controlled motion of skyrmions are essential features in order (a)
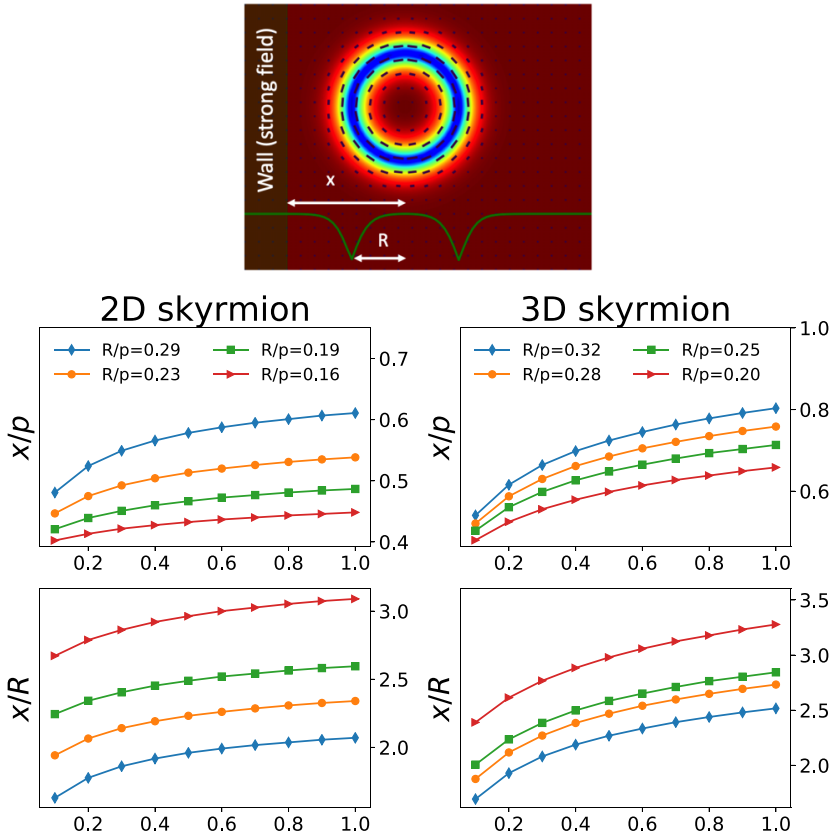

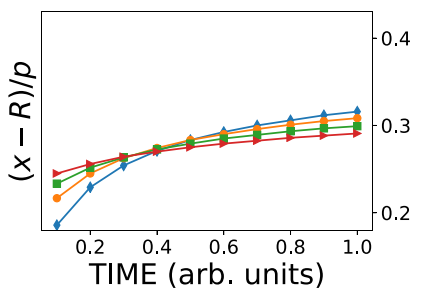

(b)

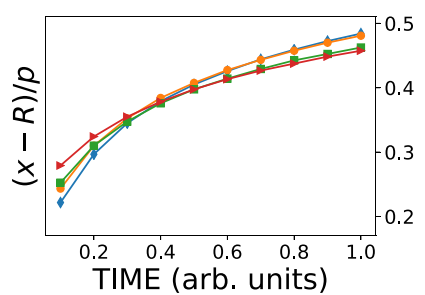

(c)
FIG. 2. (a) A skyrmion near a wall produced by a strong vertical alignment. Green line indicates the absolute value of director $n_{z}$. Here $R$ is the skyrmion radius and $x$ is the distance from skyrmion center to the wall. Panels (b) and (c) plot the ratios $x / p, x / R$, and $(x-R) / p$ as a function of time for $2 \mathrm{D}$ and $3 \mathrm{D}$ skyrmions.

to custom design materials. Surface anchoring is one way to impose boundary conditions for this purpose. Similarly, field alignment can produce regions with a desired director configuration. While surface anchoring requires preprocessing and is permanent, electric fields in principle can be turned on and off, moved, reoriented and varied with desired time dependence.

The wall mechanism described above can be used to generate repulsive regions. By the same token, attractive regions can be produced using alignment weaker than the background. The director profile of a skyrmion is approximately a vertical inner disk surrounded by a $\pi$-wall which in turn is surrounded by the vertical background. The nonvertical parts of a skyrmion will cost more energy when placed in a region with stronger vertical alignment due to larger restoring forces. Therefore, the nonvertical parts energetically prefer to overlap with weaker alignment and the vertical parts prefer to overlap with stronger alignment.

Simulation snaphots in Fig. 3 are some arrangements which generate repulsive or attractive regions. In Fig. 3(a), weaker and stronger surface anchoring regions are patterned inside a background anchoring strength of $K=2.6$ on the sur- 


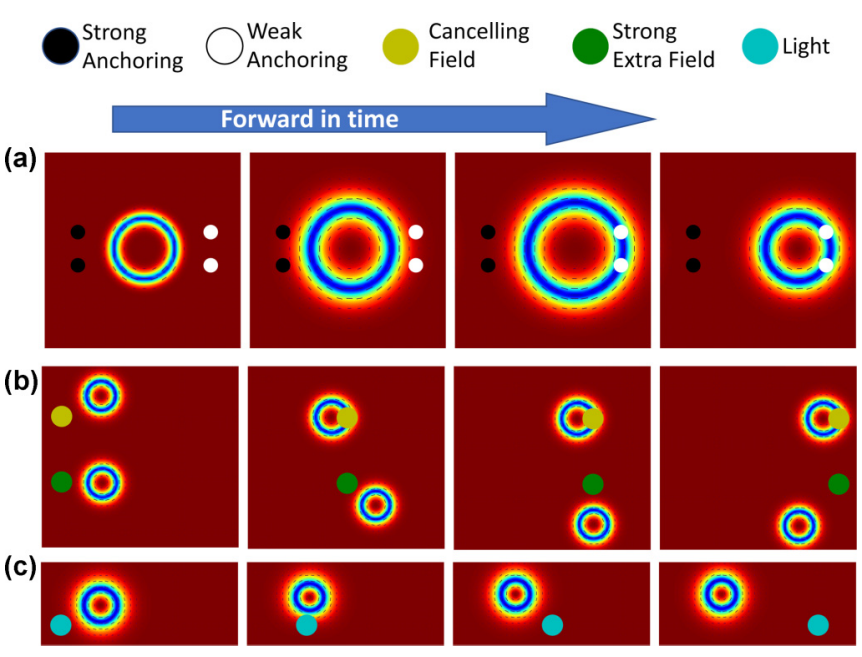

FIG. 3. Snapshots from simulations showing skyrmions near moving or stationary spots made by modifications in surface anchoring, field, or light. (a) A skyrmion is swollen by lowering the background field, so it comes in contact with nearby spots. It is then repelled by strong anchoring sites (black) and attaches itself to the weak anchoring sites (white). (b) Skyrmions are dragged by a low field spot (yellow) and repelled by a high field spot (green). (c) Skyrmions are repelled by moving light.

faces of a LC cell of thickness 15. Black circles mark regions with strong surface anchoring, $K \approx 30$, and white circles mark regions with weak surface anchoring, $K \approx 0.3$. Initially, with $\alpha=2.7$, the skyrmion is placed between these circles and then the background field is reduced so that the skyrmion swells and interacts with the black and white regions. Finally, the field is brought back to the initial value of $\alpha=2.7$ to deswell the skyrmion. In the final picture, the skyrmion ends up adhering to the weaker anchoring sites. In Fig. 3(b), a LC cell of thickness 15 with no surface anchoring is shown. The green spot denotes a region with stronger electric field of $\alpha=85$ in the $z$ direction and the yellow spot denotes a region in which the background field of $\alpha=5$ is canceled out by applying an electric field of equal magnitude in the opposite direction. Once these spots are moved toward the skyrmions, the green stronger-field spot repels and the yellow weakerfield spot attracts and drags the skyrmions. In Fig. 3(c), we show snapshots demonstrating a different mechanism, light exposure. Exposure to light chemically increases the helical pitch of some LC materials [21,27]. To model the effect of light in simulations, we reduce the natural twist $q_{0}$ by 1.5 in the region of exposure. When we move that region toward a skyrmion, the skyrmion is repelled away from light. Our simulations confirm that reduction in $q_{0}$ is a valid mechanism to generate repulsive sites realized in the experiments mentioned above. All of these mechanisms can be used alone or in combination to generate patterned repulsive and/or attractive regions. (See the SM [22] for movies of each of the above mechanisms).

Can skyrmions be combined when squeezed together? As a test, we placed skyrmions between moving walls and reduced the volume between the walls [video in SM [22] part (E)]. As the walls move closer like a trash compacter no skyrmions fused. Interestingly, as the pressure (a)

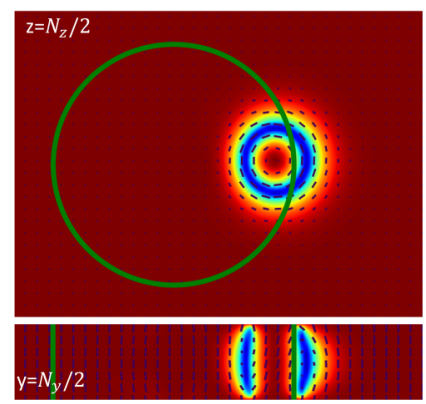

(b)

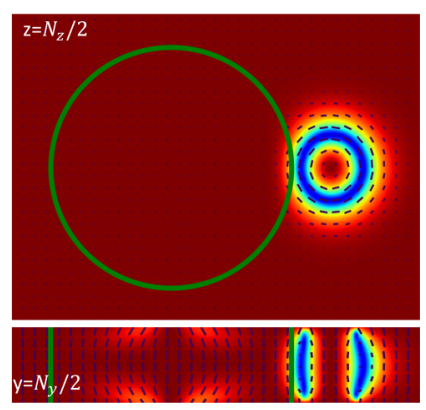

FIG. 4. Repulsive site generated by point electrodes: Oppositely charged point charges are placed at positions $N_{z} / 2 \pm H$ above and below the midplane of the LC cell, where $H=15, N_{z}=16, p=40$, $K / L S q_{0}=7.9$, and $\alpha / L S q_{0}^{2}=1.7$ with material parameters listed in the SM [22]. The green circle denotes the region with radius $R=2 H=30$ outside which the field vanishes (see the SM [22]). When the skyrmions are driven by moving the wall region (green circle) an effective wall size is experienced depending on the potential difference (the greater the potential difference the greater the size). Top panels show horizontal cross section at $z=N_{z} / 2$ and bottom panels show vertical cross section at $y=N_{y} / 2$. The potential difference (charge) is chosen such that the extra field at the midpoint of the cell is equal to (a) 1 and (b) 6 times the background field.

increases the skyrmions get smaller and smaller until they pop spontaneously.

Fringe effect and very thin walls. In the demonstrations above, we employed a uniform extra field produced in the form of a circular pillar as small as a fraction of a skyrmion which may seem experimentally difficult to achieve. In principle, the cross section of the pillar does not have to be small in order to push or drag skyrmions. However, in some cases fringe effects caused by the small electrodes generating the electric field across the LC cell will be important. Here we address the case of very small electrodes placed on both sides of the LC cell.

When a potential difference $\Delta V$ is applied between two such electrodes, charges of opposite sign and equal magnitude of $q=C \Delta V$ will accumulate at the electrodes where $C$ is the capacitance. For very small size limit, we can safely assume that they are point charges. In Fig. 4 we show how a field generated by such point charges can drive skyrmions. (The calculation of the field is shown in the SM [22].) In Fig. 4(a), a relatively weaker field is applied. In Fig. 4(b), even when the extra field is much bigger than the background field leading to obvious deviation of the directors from the vertical direction, skyrmions are still successfully driven. Comparison of Figs. 4(a) and 4(b) illustrates that the effective radius of the pillar depends on the potential difference applied across the LC cell. This feature adds another useful knob to the tool set to control skyrmions because changing the size of a repulsive (or attractive) pillar by means of varying the potential difference is much easier than changing the geometry and structure of the system and it allows dynamic control of the wall size. Furthermore, it helps overcome limitations on how small the walls can be made because we can reduce the electrode potential difference all the way to zero and achieve extremely small wall sizes. 
Discussion. We have demonstrated how electric field, surface anchoring and light can be used to generate repulsive and attractive regions in order to precisely design desired patterns and assemblies of skyrmions. Our model includes some approximations such as the widely used single elastic constant approximation and the assumption of a uniform electric field perpendicular to the confining plates. Isolated skyrmions realized in experiments are reproduced by our model with great resemblance in structure, shape and phase behavior. Therefore, while a more detailed model that includes nonuniform elastic constants or deviations from a uniform electric field will surely provide higher-order corrections, we do not think it would significantly alter the qualitative behavior. In simulations depicted in Fig. 3, idealized circular walls were employed which host sharp gradients of the electric field and the distribution of chemically modified molecules. We have shown in the section 'Fringe effect and very thin walls' that smoother gradients and nonuniform fields can reproduce the same wall effects. As for the light-exposed molecules, in reality, the circular shape of the wall region will not always be intact due to the diffusion of molecules and due to the time needed to modify newly illuminated molecules when light is moved. Thus, in experiments, diffusion and photochemical excitation and relaxation times need to be taken into account. To summarize, more detailed versions of our model may provide higher-order corrections but the current form sufficiently demonstrates the mechanisms and provides useful insight into the underlying physics.

The methods presented here can easily be modified or expanded to realize many diverse features for a variety of novel applications. In analogy to the letters or words in a book, skyrmions can be organized into artificial structures. As first steps, we have already numerically realized some useful and physics-rich applications including skyrmionic artificial spin ice [28] and dynamic switching of skyrmion lattice structures on substrates [29]. We envisage that systems whose constituents are micron-sized skyrmions can be programed to exhibit custom designed complex collective behaviors thanks to the possibility of individual creation and decimation of skyrmions at will and dynamic manipulation of obstacles, patterns, and even the way skyrmions interact with obstacles and other skyrmions. In addition to methods applied here to upright skyrmions, tilted skyrmions achieved by means of tilting or modulating background fields can add even richer features. Interesting skyrmion behaviors in the presence of field, light, and surface anchoring are continuously being discovered. Original experimental studies [19-21,30] that appeared recently also indicate that we are at the verge of significant progress in realizing functional materials whose constituents are skyrmions. We believe that the ideas presented here will inspire a new direction for the realization of designer skyrmion materials and motivate experimental and theoretical efforts aiming to investigate the underlying physics in more detail. Furthermore, similar methods and mechanisms can potentially be applied in magnetic systems as well [31-35].

Acknowledgments. The work at Los Alamos National Laboratory was carried out under the auspices of the U.S. DOE and NNSA under Contract No. DEAC5206NA25396 and supported by U.S. DOE (A.D. and A.S.). The work of J.V.S. was supported by NSF Grant No. DMR-1409658.
[1] R. P. Feynman, There's plenty of room at the bottom, Eng. Sci. 23, 22 (1960).

[2] T. H. R. Skyrme, A unified field theory of mesons and baryons, Nucl. Phys. 31, 556 (1962).

[3] S. Mühlbauer, B. Binz, F. Jonietz, C. Pfleiderer, A. Rosch, A. Neubauer, R. Georgii, and P. Böni, Skyrmion lattice in a chiral magnet, Science 323, 915 (2009).

[4] N. Nagaosa and Y. Tokura, Topological properties and dynamics of magnetic skyrmions, Nat. Nanotechnol. 8, 899 (2013).

[5] J.-I. Fukuda and S. Zumer, Quasi-two-dimensional skyrmion lattices in a chiral nematic liquid crystal, Nat. Commun. 2, 246 (2011).

[6] A. O. Leonov, I. E. Dragunov, U. K. Rößler, and A. N. Bogdanov, Theory of skyrmion states in liquid crystals, Phys. Rev. E 90, 042502 (2014).

[7] A. N. Bogdanov, U. K. Rößler, and A. A. Shestakov, Skyrmions in nematic liquid crystals, Phys. Rev. E 67, 016602 (2003).

[8] P. J. Ackerman, R. P. Trivedi, B. Senyuk, J. van de Lagemaat, and I. I. Smalyukh, Two-dimensional skyrmions and other solitonic structures in confinement-frustrated chiral nematics, Phys. Rev. E 90, 012505 (2014).

[9] S. Afghah and J. V. Selinger, Theory of helicoids and skyrmions in confined cholesteric liquid crystals, Phys. Rev. E 96, 012708 (2017).

[10] Y. Guo, S. Afghah, J. Xiang, O. D. Lavrentovich, R. L. B. Selinger, and Q.-H. Wei, Cholesteric liquid crystals in rectangu- lar microchannels: skyrmions and stripes, Soft Matter 12, 6312 (2016).

[11] P. J. Ackerman, J. van de Lagemaat, and I. I. Smalyukh, Selfassembly and electrostriction of arrays and chains of hopfion particles in chiral liquid crystals, Nat. Commun. 6, 6012 EP (2015).

[12] D. Foster, C. Kind, P. J. Ackerman, J.-S. B. Tai, M. R. Dennis, and I. I. Smalyukh, Two-dimensional skyrmion bags in liquid crystals and ferromagnets, Nat. Phys. 15, 655 (2019).

[13] R. M. Hornreich and S. Shtrikman, Field-induced hexagonal blue phases in positive and negative dielectric anisotropy systems: Phase diagrams and topological properties, Phys. Rev. A 41, 1978 (1990).

[14] A. Nych, J.-I. Fukuda, U. Ognysta, S. Zumer, and I. Musevic, Spontaneous formation and dynamics of half-skyrmions in a chiral liquid-crystal film, Nat. Phys. 13, 1215 EP (2017).

[15] L. Metselaar, A. Doostmohammadi, and J. M. Yeomans, Topological states in chiral active matter: Dynamic blue phases and active half-skyrmions, J. Chem. Phys. 150, 064909 (2019).

[16] A. Duzgun, J. V. Selinger, and A. Saxena, Comparing skyrmions and merons in chiral liquid crystals and magnets, Phys. Rev. E 97, 062706 (2018).

[17] S. Wang, M. Ravnik, and S. Žumer, Surfacepatterning generated half-skyrmion lattices in 
cholesteric blue phase thin films, Liq. Cryst. 45, 2329 (2018) .

[18] H. R. O. Sohn and I. I. Smalyukh, Electrically powered motions of toron crystallites in chiral liquid crystals, Proc. Natl. Acad. Sci. USA 117, 6437 (2020).

[19] B.-X. Li, V. Borshch, R.-L. Xiao, S. Paladugu, T. Turiv, S. V. Shiyanovskii, and O. D. Lavrentovich, Electrically driven three-dimensional solitary waves as director bullets in nematic liquid crystals, Nat. Commun. 9, 2912 (2018).

[20] Y. Shen and I. Dierking, Dynamics of electrically driven solitons in nematic and cholesteric liquid crystals, Commun. Phys. 3, 14 (2020).

[21] H. R. O. Sohn, C. D. Liu, Y. Wang, and I. I. Smalyukh, Lightcontrolled skyrmions and torons as reconfigurable particles, Opt. Express 27, 29055 (2019).

[22] See Supplemental Material at http://link.aps.org/supplemental/ 10.1103/PhysRevResearch.3.L012005 for (i) simulation videos referred to in the sections Repulsive or attractive regions and Fringe effect and very thin walls, (ii) simulation methods and parameters, and (iii) the calculation of the electric field as used in the point-electrode simulations that are discussed in the section Fringe effect and very thin walls.

[23] P. J. Ackerman, T. Boyle, and I. I. Smalyukh, Squirming motion of baby skyrmions in nematic fluids, Nat. Commun. 8, 673 (2017).

[24] I. I. Smalyukh, B. I. Senyuk, P. Palffy-Muhoray, O. D. Lavrentovich, H. Huang, E. C. Gartland, V. H. Bodnar, T. Kosa, and B. Taheri, Electric-field-induced nematic-cholesteric transition and three-dimensional director structures in homeotropic cells, Phys. Rev. E 72, 061707 (2005).

[25] G. Durey, H. R. O. Sohn, P. J. Ackerman, E. Brasselet, I. I. Smalyukh, and T. Lopez-Leon, Topological solitons, cholesteric fingers and singular defect lines in janus liquid crystal shells, Soft Matter 16, 2669 (2020).
[26] J.-S. B. Tai and I. I. Smalyukh, Surface anchoring as a control parameter for stabilizing torons, skyrmions, twisted walls, fingers, and their hybrids in chiral nematics, Phys. Rev. E 101, 042702 (2020).

[27] H. R. O. Sohn, P. J. Ackerman, T. J. Boyle, G. H. Sheetah, B. Fornberg, and I. I. Smalyukh, Dynamics of topological solitons, knotted streamlines, and transport of cargo in liquid crystals, Phys. Rev. E 97, 052701 (2018).

[28] A. Duzgun and C. Nisoli, Skyrmion spin ice in liquid crystals, arXiv:1908.03246 [cond-mat.soft] [Phys. Rev. Lett. (to be published)].

[29] A. Duzgun, C. Nisoli, C. J. O. Reichhardt, and C. Reichhardt, Commensurate states and pattern switching via liquid crystal skyrmions trapped in a square lattice, Soft Matter 16, 3338 (2020).

[30] E. Bukusoglu, M. Bedolla Pantoja, P. C. Mushenheim, X. Wang, and N. L. Abbott, Design of responsive and active (soft) materials using liquid crystals, Annu. Rev. Chem. Biomolec. Eng. 7, 163 (2016).

[31] S.-Z. Lin, C. Reichhardt, C. D. Batista, and A. Saxena, Driven Skyrmions and Dynamical Transitions in Chiral Magnets, Phys. Rev. Lett. 110, 207202 (2013).

[32] R. Tomasello, S. Komineas, G. Siracusano, M. Carpentieri, and G. Finocchio, Chiral skyrmions in an anisotropy gradient, Phys. Rev. B 98, 024421 (2018).

[33] A. O. Leonov, T. L. Monchesky, J. C. Loudon, and A. N. Bogdanov, Three-dimensional chiral skyrmions with attractive interparticle interactions, J. Phys.: Condens. Matter 28, 35LT01 (2016).

[34] A. O. Leonov, J. C. Loudon, and A. N. Bogdanov, Spintronics via non-axisymmetric chiral skyrmions, Appl. Phys. Lett. 109, 172404 (2016).

[35] S. Luo, M. Song, X. Li, Y. Zhang, J. Hong, X. Yang, X. Zou, N. Xu, and L. You, Reconfigurable skyrmion logic gates, Nano Lett. 18, 1180 (2018). 